\title{
Necesidad de reconocimiento y síndrome de selfie: un análisis relacional basado en minería de datos
}

\author{
Víctor Daniel Gil \\ Universidad Católica Luis Amigó \\ victor.gilve@amigo.edu.co
}

(Tipo de Artículo: Reflexión. Recibido el 06/02/2017. Aprobado el 16/02/2017)

\begin{abstract}
Resumen. El uso excesivo de redes sociales en el mundo está despertando la necesidad de reconocimiento en hombres y mujeres. Estar conectado la mayor parte del día a redes sociales motiva la obsesión por los autorretratos o más conocidos como "Selfies", dando origen al moderno "Síndrome de Selfie". Este trabajo presenta un modelo de minería de datos elaborado con el programa RapidMiner para el análisis de sentimientos que permitió clasificar el efecto emocional (positivo / negativo) de los comentarios publicados en 250 "Selfies" de hombres y 250 "Selfies" de mujeres en la red social Facebook. Con el modelo se concluye que existe una fuerte correlación entre la necesidad de reconocimiento y "Sindrome de Selfie", la cual varía en función del género. Según el modelo desarrollado es mayor en el femenino, $r x y=0.75$, que en el masculino, $r x y=0.61$, lo que permite afirmar que el grado de relación o de asociación entre las dos variables es buena para el género femenino a diferencia del masculino, lo que indica que las mujeres constantemente están bajo una presión social estética mucho mayor que los hombres.
\end{abstract}

Palabras clave. minería de datos, necesidad de reconocimiento, redes sociales, revisión sistemática de literatura, síndrome de selfie.

\section{Need for recognition and selfie syndrome: a relational analysis based on data mining}

\begin{abstract}
The excessive use of social networks in the world is waking the need for recognition in men and women. Be connected the greater part of the day to social networks motivates the obsession with the self-portraits or more known as "Selfies", giving rise to the modern "Selfie Syndrome". This paper presents a data mining model for the feelings analysis that allowed to classify the emotional impact (positive/negative) of the comments published in 250 "Selfies" of men and 250 "Selfies" of women in the social network Facebook. This paper concludes that there is a strong correlation between need for recognition and "Selfie Syndrome". According to the model developed is greater in the female gender, $r_{x y}=0.75$, that the male gender, $r x y=0.61$, which allows to affirm that the degree of relationship or association between the two variables is good for the female gender unlike male, which indicates that the women are constantly under aesthetics social pressure much greater than men.
\end{abstract}

Keywords. data mining, need for recognition, social networks, systematic literature review, selfie syndrome. 


\section{Introducción}

En la última década, el creciente uso de las redes sociales en el mundo está motivando a las personas a adoptar comportamientos y actitudes narcisistas, pese a que en la mayoría de países es considerado una moda. Día a día, una gran cantidad de personas comparten autorretratos, también conocidos como "Selfies", para satisfacer su necesidad de reconocimiento. Este trabajo tiene como objetivo principal desarrollar un modelo de minería de datos que permita identificar si existe una relación entre "síndrome de selfie" y necesidad de reconocimiento.

En la revisión del estado del arte se empleó la metodología de la revisión sistemática de literatura (RSL), revisión sistemática, organizada, auditable y estructurada, que busca dar respuesta a una o más preguntas de investigación tomando como base investigaciones científicas y publicaciones previas [1]. La pregunta de investigación considera fue:

P1. ¿Cuál es la relación existente entre Necesidad de reconocimiento y Síndrome de "Selfie"? Con esta pregunta se pretende identificar si existe o no alguna relación entre ambas variables.

El resto del trabajo se divide como sigue: en primer lugar se presenta una contextualización general sobre necesidad de reconocimiento y "síndrome de selfie", la metodología empleada y por último los resultados obtenidos. Se concluye que el grado de reconocimiento mutuo (comentarios y "me gusta") es más fuerte para las mujeres que para los hombres, es decir, los selfies de mujeres producen un mayor impacto y respuestas en comparación a las de los hombres.

\section{Revisión sistemática de literatura}

La revisión sistemática de la literatura (RSL) fue formulada por primera vez en el campo de la medicina con el objetivo principal de sintetizar los resultados de investigaciones médicas previas que facilitaran la identificación de temáticas para realizar futuras investigaciones. En la actualidad, la revisión sistemática de literatura es utilizada en diferentes áreas del conocimiento [2]. Según Kitchenham (2004), la RSL es un proceso de descubrimiento de nuevo conocimiento, el cual está basado en el método científico para identificar, evaluar y analizar estudios primarios para responder una o más preguntas de investigación. Las principales fases de la RSL son:

- Planificación de la revisión: Se formulan las preguntas de investigación, se seleccionan las bases de datos bibliográficas donde se realizarán las búsquedas, se define el alcance y el enfoque de estudio de la investigación [3]

- Realización de la revisión: Se generan los resultados de la búsqueda, el listado de publicaciones, registros de evaluaciones y se extrae la información de cada una de ellas [2]

- Informes sobre la revisión: Se genera un reporte técnico, journal paper o conference paper, con el objetivo de dar a conocer los resultados de la revisión [1]

La RSL supera muchas de las falencias de la revisión tradicional de literatura, las cuales hacen referencia a la formulación inadecuada o inexistencia de preguntas de investigación, la falta de criterios de inclusión y exclusión, y la falta de reproductibilidad en la búsqueda y análisis de información [3].

En la revisión se consultaron las bases de datos científicas: Scopus, IEEE Digital Library, SpringerLink, ScienceDirect, Scielo y páginas web especializadas. Las palabras clave de búsqueda utilizadas fueron: Selfie syndrome, Need for recognition, Low self esteem, Social Networks, Depression. Las ecuaciones de búsqueda utilizadas fueron:

(1) (TITLE-ABS-KEY (Need for recognition) OR (Social Networks) AND (((Selfie OR (((Esteem) OR ((Syndrome) OR (Depression))))

(2) (TITLE-ABS-KEY (Need for recognition) AND (Social Networks) AND (((Selfie) AND (((Esteem) AND ((Syndrome) AND (Depression))))

(3) (TITLE-ABS-KEY (Need for recognition) AND (Social Networks) AND (((Selfie) OR (((Esteem) AND ((Syndrome) OR (Depression))))

(4) (TITLE-ABS-KEY (Need for recognition) AND (Social Networks) AND (((Selfie) AND (((Esteem) AND ((Syndrome) OR (Depression))))

(5) (TITLE-ABS-KEY (Need for recognition) OR (Social Networks) OR (((Selfie) OR (((Esteem) OR ((Syndrome) AND (depression))))

El rango de fechas de las búsquedas estuvo comprendido desde el año 2008 hasta el 2016. Los documentos que se consideraron en la revisión fueron: journal papers, publicaciones web especializadas, capítulos de libros y artículos de conferencias. Los documentos que se excluyeron fueron resúmenes de artículos y prefacios. La Tabla 1, presenta el número de publicaciones por tipo recopiladas en la revisión:

Tabla 1. $\mathrm{N}^{\circ}$ de Publicaciones. Fuente: elaboración de los autores

\begin{tabular}{ll}
\hline Tipo & Cantidad \\
\hline Artículos & 22 \\
\hline Artículos de conferencias & 5 \\
\hline Capítulos de libros & 3 \\
\hline Publicaciones web & 5 \\
\hline Total & 35 \\
\hline
\end{tabular}

\section{Necesidad de reconocimiento, narcisismo y síndrome de selfie}

Según Richetin, Xaiz, Maravita, y Perugini (2012) la imagen corporal, el reconocimiento del cuerpo, y la autoestima son conceptos altamente conectados. El "Narcisismo" es un trastorno de la personalidad que implica la preocupación exagerada por sí mismo y sobre cómo se es percibido por los demás (Centre Can Roselló, 2012; Buffardi \& Campbell, 2008). El narcisista 
está interesado por la satisfacción de su vanidad y la admiración de sus propios atributos físicos e intelectuales (Buffardi \& Campbell, 2008; Centre Can Roselló, 2012). Según la clínica especializada en adicciones y patología dual "Centre can Roselló", una persona narcisista se caracteriza por no escuchar, ser egoísta, preocuparse excesivamente por sí misma, sentirse superior a los demás, no aceptar responsabilidades, ser explosivos, entre otros.

La palabra "Selfie" fue seleccionada como la palabra del año en el 2013 por el diccionario inglés Oxford. En términos generales hace referencia al autoretrato realizado con una cámara fotográfica [6]. El "Síndrome de Selfie" refleja una combinación de una amplia gama de trastornos neuropsiquiátricos: estrés, obsesión, necesidad de reconocimiento, comportamiento obsesivo-compulsivo, ansiedad, manía, trastorno bipolar y dismorfia corporal [7]. En una investigación realizada por la Academia Americana de Cirugía Plástica Facial y Reconstructiva concluyó que los "Selfies" han contribuido al aumento de procedimientos en personas jóvenes: cirugías de nariz, de párpados, trasplantes de cabello, entre otras [7]. En la Tabla 2, se presenta la clasificación de los tipos "Selfies" realizada por [8]:

Tabla 2. Clasificación de "Selfies". Fuente: elaboración de los autores

\begin{tabular}{ll}
\hline Nombre & Descripción \\
\hline $\begin{array}{l}\text { No make-up } \\
\text { selfies }\end{array}$ & $\begin{array}{l}\text { Con la cara lavada, al natural sin } \\
\text { maquillaje }\end{array}$ \\
\hline Uglies selfies & haciendo muecas \\
\hline Fit selfies & $\begin{array}{l}\text { mostrando la figura construida en el } \\
\text { gimnasio }\end{array}$ \\
\hline Couplies & de parejas enamoradas \\
\hline Celebrity selfies & con artistas famosos \\
\hline Power selfies & con personas influyentes \\
\hline Ego selfie & look diario \\
\hline Promo-selfie & $\begin{array}{l}\text { usadas por celebridades para mostrar } \\
\text { sus productos }\end{array}$ \\
\hline Airplane- selfie & al interior de un avión \\
\hline Beach time selfie & $\begin{array}{l}\text { piernas bronceadas, alberca, mar, } \\
\text { bikinis }\end{array}$ \\
\hline Tourist-Selfie & $\begin{array}{l}\text { crónicas de viaje a través de uno } \\
\text { mismo }\end{array}$ \\
\hline Pregnantselfie & futuras madres \\
\hline
\end{tabular}

La autoestima y la necesidad de reconocimiento pueden ser predictores de la importancia personal de Facebook Błachnio, Przepiorka, y Rudnicka (2016). Por otra parte, Marshall, Lefringhausen y Ferenczi (2015), afirman que en esta red social, las actualizaciones que tienen que ver con relaciones personales y de pareja es un indicador de baja autoestima, las relacionadas a la consecución de logros personales: ejercicio físico o dieta están asociadas a personalidades narcisistas y necesitadas de atención que buscan aprobación y refuerzo en la comunidad de Facebook. Por otra parte, las relacionadas con hijos indican personalidades escrupulosas [10].

Otras investigaciones relacionadas han demostrado que el apoyo de la comunidad en esta red social tiene beneficios psicológicos similares a los sentimientos de inclusión y pertenencia a un grupo social [11]. Sin embargo, no se puede afirmar con exactitud que las actualizaciones definan la personalidad de una persona [10]. En efecto, las actualizaciones narcisistas relacionadas con la consecución de logros personales son las que mayor grado de aceptación tienen y recaudan más "Me gusta" y comentarios, aunque algunas de esas personas en realidad desprecien dicho comportamiento, sin embargo escriben comentarios por cortesía [10]. Błachnio, Przepiórka, \& Pantic (2015), afirman que el uso excesivo de Facebook podría tener efectos perjudiciales sobre la salud mental y estar asociado con ciertos problemas psicológicos.

Algunas investigaciones han demostrado que existe una fuerte correlación entre necesidad de reconocimiento y los medios de comunicación social, ver los trabajos de Lee et al., (2014), McKinney, (2012) y Mehdizadeh, (2010) . La investigación realizada por Sheldon y Bryant (2016), muestra que existe una relación positiva entre los altos niveles de actividad social: viajar, ir a eventos deportivos, visitar a los amigos, etc., y estar motivados para usar Instagram como medio de documentación.

Tomarse "Selfies" en exceso" es un indicador de depresión, baja autoestima, falta de identidad e inseguridad [14]. La mayoría de pacientes diagnosticados con Trastorno Dismórfico Corporal (TDC) o "trastorno de fealdad imaginaria", enfermedad psiquiátrica que se manifiesta como una gran preocupación por un defecto imaginario de la apariencia, son adictos a tomarse autorretratos y a publicarlos [14]. La Universidad de Brunel en el Reino Unido, considera que mientras mayor sea el número de "Selfies" de una persona, sus relaciones afectivas son más superficiales, tienen un bajo sentido de privacidad, predisposición a la ansiedad y a la depresión [15].

Los "Selfieadictos" se toman una cantidad exagerada de autorretratos y al final seleccionan solo uno, hasta el punto de tratar de editar el mayor número de errores físicos que sea posible, pretendiendo mostrar la imagen de quién quieren que piensen que son [16], situación que puede afectar la integridad física de las personas, se ha dado el caso en que algunas personas intentan suicidarse si sus "Selfies" no reúnen un determinado número de "Likes" o "Me gusta", como sucedió con el caso del joven europeo Danny Bowman [16], situación que puede motivar a las personas a realizarse cirugías plásticas y a tomar decisiones banales.

\section{Metodología}

Se emplearon dos bases de datos que reunían los comentarios que no tenían doble sentido de 250 "Selfies" de hombres y 250 "Selfies" de mujeres y se registró el número de "Me gusta" en cada uno de ellos. La base de datos estaba conformada por dos columnas, la primera contenía el tipo de sentimiento; positivo o negativo, la segunda el comentario del "Selfie". En cada una de las bases de datos se clasificaron 70 comentarios, los 180 comentarios restantes fueron clasificados por el modelo. 
En total se emplearon 500 comentarios. Los comentarios de los selfies para el género femenino fueron realizados por más mujeres que hombres al igual que los comentarios para el género masculino. En la Tabla 3, se presenta la estructura de las bases de datos:

Tabla 3. Estructura Base de Datos. Fuente: elaboración de los autores

\begin{tabular}{ll}
\hline Sentimiento & \multicolumn{1}{c}{ Comentario } \\
\hline Positivo & Divinos ojos, das envidia \\
\hline Positivo & Espectacular, divina !! \\
\hline$?$ & Espero distinguirte pronto \\
\hline$?$ & $\begin{array}{l}\text { No me regalas tu sobrado o tu esposa es } \\
\text { celosa }\end{array}$ \\
\hline Negativo & Así es siempre mentiras y más mentiras \\
\hline$?$ & Que ruda tomándose esas fotos! \\
\hline Negativo & $\begin{array}{l}\text { Yo le regalo mi gripa, así usted descansa y } \\
\text { yo a la calle... }\end{array}$ \\
\hline Positivo & muy lindo torso, felicitaciones \\
\hline$\ldots$ & $\ldots$
\end{tabular}

El modelo fue desarrollado en el software RapidMiner, el cual permite ejecutar procesos de análisis de datos mediante el encadenamiento de operadores a través de un entorno gráfico [17]. Es utilizado en investigación, educación, capacitación, creación rápida de prototipos y en aplicaciones empresariales [17]. A continuación, se describen los principales operadores del modelo los cuales están conectados en un orden lógico y secuencial [18] citado por [19]:

- Pre - proceso: introduce un proceso dentro de otro. Un subproceso puede ser considerado como una unidad pequeña de un proceso. Una vez que se completa la ejecución de un subproceso, el flujo se devuelve al proceso inicial.

- Multiplicador: copia el objeto de entrada a todos los puertos de salida que están conectados sin modificarlo.

- Remoción de atributos inútiles: elimina atributos inútiles de la base de datos. Los umbrales para atributos inútiles son especificados por el usuario

- Filtro de entrenamiento: es el operador raíz más exterior al operador de cada proceso.

- Curva de aprendizaje: itera sobre cada subproceso todas las combinaciones posibles de parámetros definidos.

- Árbol de decisión: genera un árbol de decisión para la clasificación de datos nominales y numéricos.

- Modelo final: devuelve el objeto de salida especificado de la colección total de datos.

- Rama: consta de dos sub-procesos pero ejecuta sólo un subproceso a la vez dependiendo de la condición. Es similar a la declaración "if-then-else", donde una de las dos opciones se selecciona en función de los resultados de la condición especificada.

Todos los procesos de descubrimiento de nuevo conocimiento, así como los modelos de minería de datos para la identificación de patrones en grandes volúmenes de información, requieren la compresión exhaustiva de la manera de cómo se van a aplicar éstas técnicas, métodos y herramientas en la vida real [20]. La Figura 1, se presenta el diagrama estructural del modelo desarrollado.

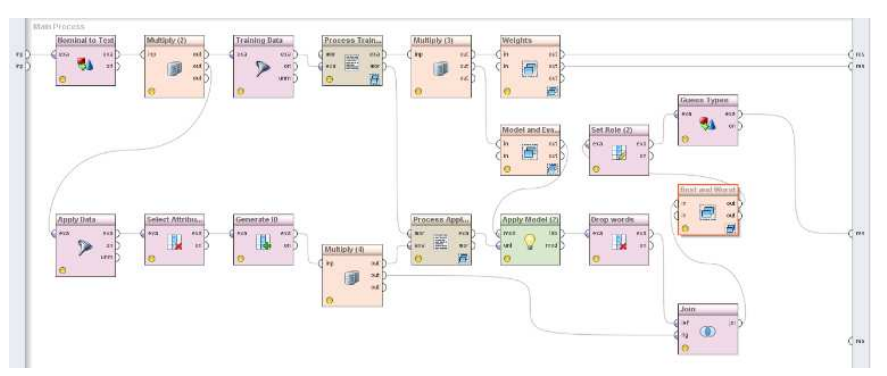

Figura 9. Diagrama estructural del modelo desarrollado

El modelo desarrollado clasifica los comentarios de cada uno los "Selfies" que no fueron clasificados en la base de datos de entrada (positivos / negativos), identifica las palabras más importantes de cada comentario que determinan los sentimientos positivos o negativos y por último asigna una puntuación. En la Tabla 4, se presenta un cuadro resumen del resultado final arrojado por el modelo. En la primera columna se clasifican los comentarios como positivos o negativos, en la segunda columna el puntaje de cada comentario en una escala de 0 a 100, donde una mayor puntuación significa un sentimiento más positivo y en la tercera el comentario de cada "Selfie".

Tabla 4. Resultado del Modelo. Fuente: elaboración de los autores

\begin{tabular}{|c|c|c|}
\hline Sentimiento & Puntaje & Comentario \\
\hline Positivo & 80 & Hermosa creación de Dios \\
\hline Positivo & 78 & $\begin{array}{l}\text { Ojitos. Gracias por darle } \\
\text { alegrías a mi vida al poder } \\
\text { contemplar tu belleza }\end{array}$ \\
\hline Positivo & 78 & Pareces una estrella de plata \\
\hline Positivo & 77 & $\begin{array}{l}\text { Divina, ese color de pelo queda } \\
\text { divino }\end{array}$ \\
\hline Positivo & 76 & Hermoso, pareces un príncipe \\
\hline Positivo & 75 & Bellezas las dos, radiantes \\
\hline Positivo & 75 & $\begin{array}{l}\text { Hermosa, como todos los } \\
\text { felinos... }\end{array}$ \\
\hline Positivo & 74 & Simplemente, Hermosa \\
\hline : & : & : \\
\hline
\end{tabular}

\section{Resultados}

La Figura 2 y Figura 3 presentan las palabras más importantes de los comentarios que fueron clasificados como sentimientos positivos y negativos en la base de datos de los hombres. En esta se pueden identificar tópicos y términos relevantes que frecuentemente son utilizados cuando las personas expresan sentimientos positivos. 


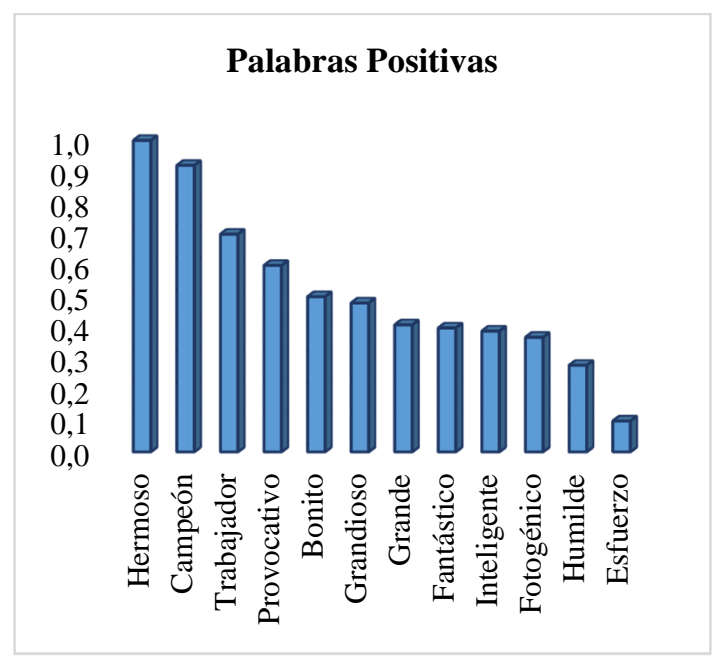

Figura 2. Peso Vs Palabra

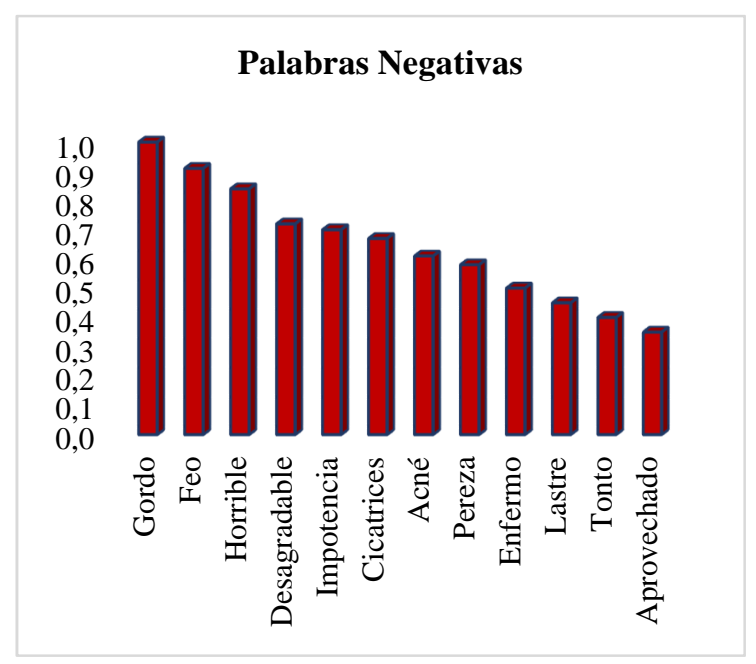

Figura 3. Peso Vs Palabra

La Figura 4 y Figura 5 presentan las palabras más importantes de los comentarios que fueron clasificados como sentimientos positivos y negativos en la base de datos de las mujeres.

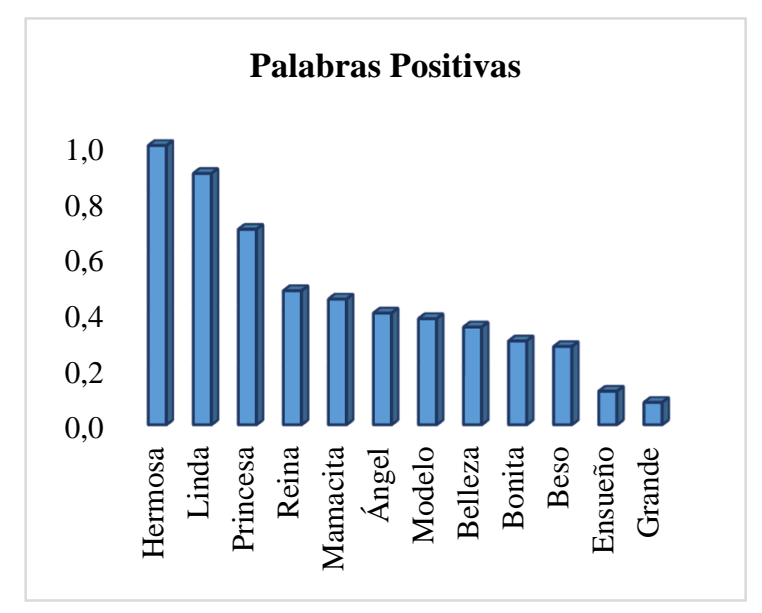

Figura 4. Peso Vs Palabra

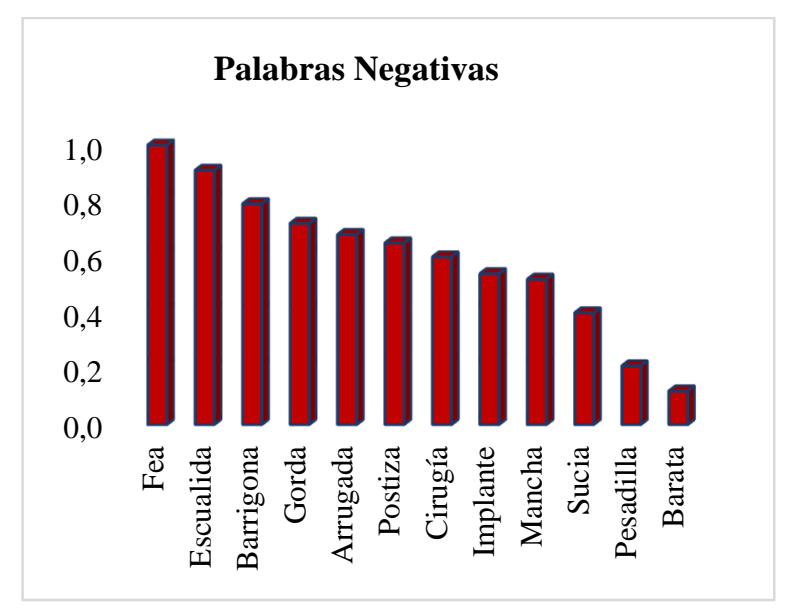

Figura 5. Peso Vs Palabra

Para el caso de los hombres el modelo clasificó108 comentarios positivos y 72 negativos, en el caso de las mujeres 143 positivos y 37 negativos. Se determinó el coeficiente de correlación de Pearson entre las puntuaciones arrojadas por el modelo y el número de "Me gusta" de cada "Selfie" en las dos bases de datos. Ver ecuación (1):

$$
r_{x y}=\frac{\sum x y}{\sqrt{x^{2}} \sqrt{y^{2}}}
$$

donde $r_{X Y}$ es la covarianza de $(\mathrm{X}, \mathrm{Y}), \sqrt{x^{2}}$ es la desviación típica de la variable $X$ y $\sqrt{y^{2}}$ es la desviación típica de la variable $Y$.

Se debe aclarar que el coeficiente de Pearson nos ofrece información del grado de asociación o de relación entre dos variables, pero no señala causalidad. Es decir, no informa sobre que variable es causa y cuál es la que sufre la influencia de otra. En la Tabla 5, se presenta los resultados del coeficiente de correlación de Pearson para el género masculino, en la Tabla 6 , los resultados para el género femenino:

Tabla 5. Coeficiente Pearson Hombres. Fuente: elaboración de los autores

\begin{tabular}{|c|c|c|}
\hline & Puntaje & $\mathrm{N}^{\circ}$ de "Me gusta" \\
\hline Puntaje & 1 & \\
\hline $\mathrm{N}^{\circ}$ "Me gusta" & 0,6154 & 1 \\
\hline \multicolumn{3}{|c|}{$\begin{array}{l}\text { Tabla 6. Coeficiente Pearson Mujeres. Fuente } \\
\text { elaboración de los autores }\end{array}$} \\
\hline & Puntaje & $\mathrm{N}^{\circ}$ de "Me gusta" \\
\hline Puntaje & 1 & \\
\hline $\mathrm{N}^{\circ}$ "Me gusta" & 0,7565 & 1 \\
\hline
\end{tabular}

El valor del coeficiente de correlación de Pearson es positivo en ambos casos, es decir, existe una correlación positiva y directa entre las dos variables analizadas. Sin embargo, dicha correlación varía en función del sexo, según el modelo desarrollado existe una mayor correlación en el género femenino, $r_{x y}=0.75$, que en el masculino, $r_{x y}=0.61$, lo que permite afirmar que las 
mujeres constantemente están bajo una presión estética social mucho más fuerte que los hombres.

\section{Conclusiones}

A pesar de que la mayoría de los sitios de redes sociales en línea como Facebook, Instagram y Twitter fueron lanzados en la última década, actitudes y comportamientos narcisistas han aumentado a nivel mundial debido a la dinámica que se mueve al interior de las mismas, lo que lleva a las personas a sobreestimar sus habilidades y tener una excesiva necesidad de admiración y reconocimiento externo.

La necesidad de reconocimiento es una necesidad humana ligada al bienestar psicológico y como todo, los seres humanos lo buscan entre sus pares de muchas formas. La constante necesidad de exponer la vida personal a través de autorretratos es una señal de baja autoestima e inseguridad que pone en evidencia la necesidad de ser aceptados por los demás.

\section{Referencias}

[1] B. Kitchenham, "Procedures for Performing Systematic Reviews," 2004

[2] M. Staples and M. Niazi, "Experiences using systematic review guidelines," J. Syst. Softw., vol. 80, no. 9, pp. 1425-1437, Sep. 2007.

[3] B. Kitchenham and P. Brereton, "A systematic review of systematic review process research in software engineering," Inf. Softw. Technol., vol. 55, no. 12, pp. 2049-2075, Dec. 2013.

[4] J. Richetin, A. Xaiz, A. Maravita, and M. Perugini, "Self-body recognition depends on implicit and explicit self-esteem," Body Image, vol. 9, no. 2, pp. 253-260, 2012.

[5] Centre Can Roselló, "El síndrome Selfie: de la moda al narcisismo," 2012. .
Ingenierías USBMed, Vol. 8, No. 1, Enero-Junio 2017

[6] L. Qiu, J. Lu, S. Yang, W. Qu, and T. Zhu, "What does your selfie say about you?" Comput. Human Behav., vol. 52, pp. 443-449, Nov. 2015.

[7] R. Rese, "Selfie syndrome: An Infectious Gift of IT to Health Care," vol. 2 , no. $4,2015$.

[8] C. Gonzalez, "14 tipos de "selfies," Merca2.0 Mercadotecnia publicidad y medios, 2014.

[9] A. Błachnio, A. Przepiorka, and P. Rudnicka, "Narcissism and selfesteem as predictors of dimensions of Facebook use," Pers. Individ. Dif., vol. 90, pp. 296-301, 2016.

[10] T. C. Marshall, K. Lefringhausen, and N. Ferenczi, "The Big Five, selfesteem, and narcissism as predictors of the topics people write about in Facebook status updates,” Pers. Individ. Dif., vol. 85, pp. 35-40, Oct. 2015.

[11] C. Valentini, "Is using social media 'good' for the public relations profession? A critical reflection,” Public Relat. Rev., vol. 41, no. 2, pp. 170-177, Jun. 2015

[12] A. Błachnio, A. Przepiórka, and I. Pantic, "Internet use, Facebook intrusion, and depression: Results of a cross-sectional study," Eur. Psychiatry, vol. 30, no. 6, pp. 681-684, 2015.

[13] P. Sheldon and K. Bryant, "Instagram: Motives for its use and relationship to narcissism and contextual age," Comput. Human Behav., vol. 58, pp. 89-97, 2016.

[14] W. Patterson, O. Bienvenu, P. Chodynicki, C. Janniger, and R. Schwartz, "Trastorno dismórfico corporal," Int. J. Dermatol., vol. 40, no. 11, pp. 688-690, 2001.

[15] J. Mendoza, "De la moda al narcisismo," Revista Multimedia para Estudiantes, 2015.

[16] A. Molloy, "'Selfie obsessed' teenager Danny Bowman suicidal after failing to capture "the perfect selfie," Independent, 2014. .

[17] Wikipedia, "RapidMiner," 2015.

[18] RapidMiner, "RapidMiner Studio Manual." New York, pp. 31-39, 2014.

[19] V. Gil, "Data Mining Applied to the Detection of Desertion in Young Offenders," Ing. USBMed, vol. 7, no. 2, pp. 61-66, 2016.

[20] M. Manies y U. Nikual. La elicitación de requisitos en el context de un proyecto software.Revista Ingenierias USBMed, Vol. 2, No. 2, Jul-Dic 2011. 